\title{
Heterogeneidad del color en formulaciones de merkén elaboradas a partir de ecotipos de ají (Capsicum annuum L.) cv. "Cacho de cabra"
}

\author{
Heterogeneity in color of merkén formulations prepared from ecotypes \\ of chili pepper (Capsicum annuum L.) cv. "Cacho de cabra" \\ Gina N. Leonelli Cantergiani ${ }^{*}$, Christián M. Díaz Becerra ${ }^{1}$, Ricardo M. Tighe Neira1, \\ Claudia G. Castillo Rubio ${ }^{1}$,Fernando L. Pardo Díaz ${ }^{2}$, Inès Birlouez-Aragon ${ }^{3}$
}

\begin{abstract}
RESUMEN
En el presente trabajo se evaluó la heterogeneidad del color en cinco muestras de merkén formuladas a partir de ecotipos de ají, Capsicum annuum L., cv. "Cacho de cabra" cultivados en la Región de La Araucanía y Región del Maule, Chile. La evaluación se realizó mediante la extracción y cuantificación del contenido de carotenoides. Las variables evaluadas en la cuantificación de carotenoides fueron carotenoides rojos $(\mathrm{R})$ y carotenoides amarillo-naranja (A). Además, se utilizó el sistema de color Cielab donde se evaluaron las coordenadas de luminosidad $\left(\mathrm{L}^{*}\right)$, componente verde-rojo $\left(\mathrm{a}^{*}\right)$, componente azul-amarillo $\left(\mathrm{b}^{*}\right)$, cromatismo $\left(\mathrm{C}^{*}\right)$ y tonalidad $\left(\mathrm{H}^{*}\right)$. La preparación que presentó mayor contenido de carotenoides $(\mathrm{R})$ y (A) fue el Merkén UCT005, del mismo modo esta formulación mostró los valores más altos frente a las variables $\mathrm{L}^{*}, \mathrm{a}^{*}, \mathrm{~b}^{*}$ y $\mathrm{C}^{*}$. La formulación Merkén UCT003 fue la que obtuvo un valor más alto para $\mathrm{H}^{*}$. Dado que el color es un índice de calidad, es recomendable el ají cv. "Cacho de cabra" de la Región de La Araucanía para una formulación óptima de merkén desde el punto de vista del color, ya que mostró tener un alto contenido de carotenoides.
\end{abstract}

Palabras clave: Capsicum, merkén, carotenoides, ají "Cacho de cabra".

\begin{abstract}
The study was conducted to evaluate the heterogeneity of color in samples of merkén produced from ecotypes of pepper, Capsicum annuum L. cv. "Cacho de cabra" cultivated in the Araucania and Maule Regions of Chile. Color assessment was made by the quantification of carotenoids, especifically red carotenoids $(R)$ and yellow-orange carotenoids $(A)$. Moreover, we used the color system Cielab to evaluate the coordinates of lightness $\left(L^{*}\right)$, green-red component $\left(a^{*}\right)$, blue-yellow component $\left(b^{*}\right)$, chroma $\left(C^{*}\right)$ and hue $\left(H^{*}\right)$. The formulation that showed the highest content of carotenoids $(R)$ and $(A)$ was Merkén UCT005, in addition this formulation had highest value in the variables $L^{*}, a *, b *$ and $C *$. Merkén UCT003 obtained the highest value for $H^{*}$. Therefore, the pepper cv. "Cacho de cabra" from the Araucanía Region is recommended for optimum merkén formulation from the standpoint of color. Since the color is a quality index, it is advisable to use pepper cv. "Cacho de cabra" from the Araucanía Region for merkén optimal formulation from the standpoint color: this variety also proved to have a higher content of carotenoids.
\end{abstract}

Key words: Capsicum, merkén, carotenoids, "Cacho de cabra” pepper.

\section{Introducción}

El merkén es un condimento elaborado artesanalmente por la etnia mapuche de la Región de La Araucanía, en base a ecotipos locales de ají rojo "Cacho de cabra" (Capsicum annuum L.), cuyos frutos son secados, ahumados, tostados y molidos y mezclados con sal y otros condimentos, como semillas de cilantro tostadas y molidas (Thomet y Sepúlveda, 2006). Anualmente, se transan a nivel nacional $9.000 \mathrm{t}$ de ají, y de este total, el cultivar "Cacho de cabra" corresponde aproximadamente a $1.500 \mathrm{t}$, de las cuales entre $3 \%$ y $6 \%$ son destinadas a la elaboración de merkén (INE, 2008).

El color externo de los alimentos, a través de la experiencia, la educación y alguna componente

\footnotetext{
1 Escuela de Agronomía, Facultad de Recursos Naturales, Universidad Católica de Temuco, Casilla 15-D, Rudecindo Ortega 02950, Temuco, Chile. E-mail: ginalc@uct.cl

2 Universidad de La Frontera, Avenida Francisco Salazar 01145, Temuco, Chile.

3 Spectralys Innovation, Parc d'Activités Biocitech 102, Avenue Gastón Roussel 93230, Romainville, París, Francia.

* Autor para correspondencia.
}

Fecha de Recepción: 01 Octubre, 2010.

Fecha de Aceptación: 18 Marzo, 2011. 
innata, informa sobre el estado higiénico-sanitario, el valor nutricional y además anticipa y proporciona sensaciones de otras características sensoriales como el olor y sabor. Así, el color se convierte en un índice de calidad e indica el deterioro de la misma (Mínguez-Mosquera et al., 2005). El intenso color rojo del ají maduro y sus productos procesados se debe principalmente a la biosíntesis de los pigmentos carotenoides, capsantina y capsorrubina (Curl, 1962; Davies et al., 1970), siendo estos isoprenoides $\mathrm{C}_{40}$ que contienen nueve dobles enlaces conjugados en una cadena poliénica central con diferentes grupos terminales $(\beta, \epsilon, \kappa$, 3-hydroxy-5,6-epóxido) que cambian las propiedades cromóforas de cada pigmento, permitiéndoles ser clasificados en dos familias isocrómicas, roja $(R)$ y amarilla (A) (García et al., 2007). La fracción roja contiene principalmente capsantina, capsantina-5,6epóxido y capsorrubina, junto con otros carotenoides minoritarios. La fracción amarilla comprende el resto de los pigmentos, principalmente zeaxantina, violaxantina, anteraxantina, $\beta$-criptoxantina, $\beta$-caroteno y cucurbitaxantina $A$, que corresponden a los precursores biosintéticos de la fracción roja (Hornero-Méndez et al., 2000).

La temperatura, intensidad luminosa y tiempo de secado a que se exponen los frutos de C. апnиит son variables que pueden generar aumento o disminución en la concentración de carotenoides (MínguezMosquera y Gandul-Rojas, 1994; Gómez-Ladrón y Pardo-González, 1996). La oxidación, la causa principal de las pérdidas de carotenoides, depende del oxígeno disponible, los carotenoides involucrados, la luz, calor, metales, enzimas y peróxidos que estimulan la oxidación que es inhibida por los antioxidantes tales como tocoferoles (vitamina E) y ácido ascórbico (vitamina C) (Biacs et al., 1992). Otros factores que afectan la tonalidad son la adición de $\mathrm{K}_{2} \mathrm{CO}_{3}$ o bien oleato de etilo al $2 \%+$ $\mathrm{NaOH}$ al $2 \%+4 \% \mathrm{~K}_{2} \mathrm{CO}_{3}$ a soluciones pretratantes previo al secado del ají, ya que el primero tiende a aumentar la fracción de color amarilla a diferencia del segundo, que produce una disminución de los valores de los componentes rojo y amarillo (Ergünes y Tarhan, 2006).

Debido a que el merkén es un condimento no estandarizado con creciente volumen de producción y exportación, el propósito en el presente estudio fue evaluar el color del producto merkén como índice de calidad, mediante la extracción y cuantificación de la porción de carotenoides de los grupos rojo (R) y amarillo-naranja (A) en diferentes formulaciones de merkén preparadas a partir de ecotipos de ají cv. "Cacho de cabra".

\section{Materiales y Métodos}

\section{Muestras de merkén}

Las diferentes formulaciones de merkén (Cuadro 1) fueron elaboradas y analizadas en el Laboratorio de Alimentos de la Escuela de Agronomía de la Universidad Católica de Temuco en diciembre de 2007. El ají utilizado en las formulaciones provino de distintas unidades productivas de la Región de La Araucanía ubicadas en las comunas de Lumaco, Los Sauces, Purén y Angol, y también de la Región del Maule. Las semillas de cilantro (Coriandrum sativum L.) al igual que la sal común utilizadas en las formulaciones de merkén fueron adquiridas en el comercio local.

Cuadro 1. Formulaciones de merkén en base a peso seco.

\begin{tabular}{|c|c|c|c|c|}
\hline \multirow[b]{2}{*}{ Formulación } & \multicolumn{2}{|c|}{ C. апnиит (\%) } & \multirow{2}{*}{$\begin{array}{c}\text { C. sati- } \\
\operatorname{vum}(\%)\end{array}$} & \multirow[b]{2}{*}{ Sal (\%) } \\
\hline & $\begin{array}{l}\text { R. del } \\
\text { Maule }\end{array}$ & $\begin{array}{l}\text { R. de La } \\
\text { Araucanía }\end{array}$ & & \\
\hline Merkén UCT001 & --- & 50 & 25 & 25 \\
\hline Merkén UCT002 & 40 & 10 & 25 & 25 \\
\hline Merkén UCT003 & 50 & --- & 25 & 25 \\
\hline Merkén UCT004 & 60 & --- & 30 & 10 \\
\hline Merkén UCT005 & --- & 60 & 25 & 15 \\
\hline
\end{tabular}

\section{Extracción de los grupos de carotenoides rojos (R) y amarillo-naranja (A)}

Para extraer los grupos de carotenoides $(\mathrm{R})$ y (A) se pesaron 0,5 g de cada formulación, con tres repeticiones, secadas previamente en una estufa (Memmert) durante $24 \mathrm{~h}$ a $40{ }^{\circ} \mathrm{C}$ y molidas como polvo fino de tamaño de malla $0,5 \mathrm{~mm}$ en un molino eléctrico (Fekete et al., 1976).

Cada formulación se mezcló con $20 \mathrm{~mL}$ de acetona en frascos de vidrio de $100 \mathrm{~mL}$, que fueron agitados durante $10 \mathrm{~min}$ en un shaker (Jeiotech modelo SK-300) a $125 \mathrm{rpm}$. Posteriormente, los frascos fueron dejados en reposo por $30 \mathrm{~min}$ cubiertos con papel aluminio para luego retirar el sobrenadante, repitiendo la extracción con el residuo, obteniendo un segundo sobrenadante. Se juntaron los dos sobrenadantes y luego de filtrar en papel 
filtro Wathman $\mathrm{N}^{\circ} 2$ la solución fue aforada en un matraz de $50 \mathrm{~mL}$. Una alícuota de $1 \mathrm{~mL}$ de cada extracto se diluyó en $4 \mathrm{~mL}$ de acetona leyendo la absorbancia en un espectrofotómetro (Milton Roy Modelo Spectronic $20 \mathrm{D}^{+}$) a $450 \mathrm{~nm}$ para los carotenoides del grupo amarillo-naranja (A) y $508 \mathrm{~nm}$ para los carotenoides del grupo rojo (R) (Fekete $e t$ al., 1976; Haspel-Horvatovic y Horickova, 1976; Méndez-Trujillo et al., 2005).

El contenido de los grupos de carotenoides se expresó de acuerdo a la siguiente fórmula:

$$
\mathrm{C}=\mathrm{ax} \mathrm{b}^{-1} \quad(\text { Ecuación } 1)
$$

Donde "C es el contenido de carotenoides rojos (R) o carotenoides amarillo-naranja (A) en $\mathrm{U} \mathrm{g}^{-1}$ (Unidades de absorbancia / gramo de muestra seca); "a" corresponde a la absorbancia del extracto y "b", es el peso seco de la muestra en gramos (MéndezTrujillo et al., 2005).

\section{Evaluación del color mediante el sistema de color CIELAB}

Las cinco formulaciones de merkén fueron colocadas en placas de petri de vidrio transparente, para capturar la imagen. Se utilizó una lámpara con una ampolleta corriente de luz blanca de $100 \mathrm{~W}$ para iluminar la muestra, alumbrando directamente sobre ella.

\section{1) Adquisición de la imagen}

En la captura de imagen se utilizó una cámara digital (Olympus modelo Camedia C-7070 wide 7,1 megapixel), la cual previamente fue calibrada con placas de referencia de color blanco, verde y amarillo. Luego, la cámara fue puesta sobre la parte superior de una estructura metálica bien sostenida al momento de capturar la imagen. Posteriormente, las imágenes fueron obtenidas utilizando un fondo blanco opaco bajo los siguientes ajustes: modo de disparo manual, velocidad de obturación (Tv) $1 / 13$, valor de abertura del lente (Av) 4.0, modo de medición evaluativo, velocidad ISO 50, distancia focal de 6,6 mm, sin zoom, flash automático, máxima resolución (7,1 megapíxeles), calidad superfina, balance de blancos automático, modo AF único, formato JPEG, calidad 12 (Mendoza y Aguilera, 2004). Se obtuvieron tres imágenes de cada muestra.

\section{2) Procesamiento de imágenes y análisis de color}

Las imágenes capturadas fueron analizadas en Adobe ${ }^{\circledR}$ Photoshop ${ }^{\circledR}$ versión 7.0, el primer paso previo al procesamiento de la imagen fue seleccionar el modo de color CIELAB. Posteriormente, la imagen de la muestra fue separada de su fondo negro opaco, utilizando la herramienta "varita mágica". Luego, fue activada la ventana "Histograma" para obtener los parámetros de color en términos de píxeles (L, a, b), y los parámetros de color en términos de $\mathrm{L}^{*} \mathrm{a} * \mathrm{~b} *$ fueron obtenidos mediante las siguientes ecuaciones:

$$
\mathrm{L}^{*}(\text { luminosidad })=(\mathrm{L} / 255) 100
$$

(Ecuación 2)

$\mathrm{a}^{*}($ componente verde-rojo $)=(240 \mathrm{a} / 255)-120$

(Ecuación 3)

$\mathrm{b}^{*}($ componente azul-amarillo $)=(240 \mathrm{~b} / 255)-120$

(Ecuación 4)

Matemáticamente, las variables $\mathrm{C}^{*} \mathrm{y} \mathrm{H}^{*}$ se obtienen de la transformación de las coordenadas cartesianas $a^{*} y^{*}$ a las correspondientes coordenadas polares mediante las ecuaciones informadas por Calvo y Durán (1997):

$$
\mathrm{C}^{*}(\text { cromatismo })=\left(\mathrm{a}^{* 2}+\mathrm{b}^{* 2}\right)^{1 / 2} \text {. }
$$

(Ecuación 5)

$$
\mathrm{H}^{*}(\text { tonalidad })=\tan -1(\mathrm{~b} * / \mathrm{a} *)
$$

(Ecuación 6)

\section{Análisis Estadístico}

Las variables (A), (R) fueron analizadas mediante ANDEVA y el test de comparaciones múltiples de Tukey $(p \leq 0,05)$, mientras que las variables $\mathrm{L}^{*}, \mathrm{a}^{*}$, $\mathrm{b}^{*}, \mathrm{C}^{*} \mathrm{y} \mathrm{H}^{*}$ fueron procesadas con el test Welch y test de comparaciones múltiples de Tamhane, HSD de Tukey y Games Howell, utilizando el programa SPSS ${ }^{\circledR}$ para Windows versión 15.0 en ambas metodologías.

\section{Resultados y Discusión}

El contenido de carotenoides del grupo amarillo-naranja (A) fue superior al grupo rojo (R) en todas las formulaciones (Cuadro 2), existiendo 
diferencias significativas en el valor de las medias $(\mathrm{p} \leq 0,05)$ para las formulaciones Merkén UCT003 y Merkén UCT005 como también para las formulaciones Merkén UCT001 con Merkén UCT002, tanto para la variable (A) como para la variable (R), lo que indica que el diferente contenido de carotenoides tiene relación con la procedencia del ají en la formulación de la muestra, como también de la capacidad para la síntesis de carotenoides y retención de los pigmentos de la clorofila en cada variedad de Capsicum en la etapa de plena madurez (Collera-Zúñiga et al., 2005).

Un aspecto no evaluado en este trabajo que puede incidir en el contenido de carotenoides en merkén ha sido reportado por Biacs et al. (1992), donde las semillas del cultivar de ají F-03 del tipo picante presentaron el nivel más alto del antioxidante $\alpha$-tocoferol y el producto molido de este cultivar mostró la menor degradación de carotenoides durante el almacenamiento. Los mismos autores señalan que la adición de tocoferol y ácido ascórbico redujo la pérdida del color durante el almacenamiento, siendo el ácido ascórbico el más efectivo.

El Cuadro 2 muestra que no existen diferencias significativas para el valor de las medias $(p>0,05)$ entre las formulaciones Merkén UCT001 y Merkén UCT005 elaboradas con ají de la Región de La Araucanía, tanto para la variable (A) como para la variable $(\mathrm{R})$, presentando los contenidos más altos en ambas variables. Un estudio previo realizado por García et al. (2007) señala que la acción del calor y humo resultante de la combustión de madera de encina o de hojarasca de roble, bajo un sistema de secado tradicional con flujo de aire vertical, le confiere a la páprika características de sabor ahumado y gran estabilidad del color. Por esta razón es importante la selección de las condiciones adecuadas de secado, ya que éstas son necesarias para reducir al mínimo el estrés térmico durante el exceso de secado y de esta forma permitir el mantenimiento de los compuestos que determinan la calidad del producto (Ramesh et al., 2001). Por otra parte, factores genéticos y estado de madurez de los frutos también pueden variar la composición y concentración de carotenoides en el producto (Russo y Howard, 2002; Deepa et al., 2007).

Los valores más bajos en los contenidos para ambos grupos de carotenoides correspondieron a las formulaciones Merkén UCT002 y Merkén UCT003, preparadas en base a ají cv. "Cacho de cabra" de la Región del Maule, sin presentar diferencias
Cuadro 2. Contenido ( $\mathrm{U} \mathrm{g}^{-1}$ de muestra seca) de carotenoides de los grupos (A) y (R) en relación a la formulación del producto.

\begin{tabular}{lcc}
\hline \multirow{2}{*}{ Formulación } & \multicolumn{2}{c}{ Contenido (U g ${ }^{-1}$ de muestra seca) } \\
\cline { 2 - 3 } Grupo (A) & Grupo (R) \\
\hline Merkén UCT001 & $8,5681^{\mathrm{a}} \pm 0,36857$ & $4,3473^{\mathrm{a}} \pm 0,19576$ \\
Merkén UCT002 & $5,2852^{\mathrm{b}} \pm 0,05694$ & $2,6825^{\mathrm{b}} \pm 0,01856$ \\
Merkén UCT003 & $4,5335^{\mathrm{b}} \pm 0,13809$ & $2,2933^{\mathrm{b}} \pm 0,07301$ \\
Merkén UCT004 & $5,8522^{\mathrm{ab}} \pm 0,03439$ & $2,9394^{\mathrm{ab}} \pm 0,02595$ \\
Merkén UCT005 & $9,2199^{\mathrm{a}} \pm 0,09112$ & $4,5784^{\mathrm{a}} \pm 0,06039$ \\
\hline
\end{tabular}

Letras distintas en las columnas difieren significativamente entre tratamientos según Test de Tukey $(\mathrm{p} \leq 0,05)$. Valores en base a la media.

Grupo (A): Carotenoides amarillo-naranja; grupo (R): carotenoides rojos.

significativas $(\mathrm{p}>0,05)$ entre los respectivos contenidos (Cuadro 2). La variación del contenido de carotenoides en merkén podría explicarse considerando un estudio realizado por García et al. (2007), quienes informan que la concentración de pigmentos es más alta en el pericarpio de los frutos de ciertos cultivares de $C$. аппиит, a diferencia de la páprika donde el contenido de carotenoides es menor debido al efecto de dilución de las semillas. También se ha reportado que el nivel de carotenoides en los frutos de Capsicum depende fundamentalmente de la maduración del fruto, y esta dependencia puede variar entre los cultivares (Topuz y Ozdemir, 2007). Además, su contenido está relacionado con la variedad, madurez y factores tecnológicos (Deepa et al., 2007).

El Cuadro 3 muestra los valores medios de las coordenadas cromáticas $\mathrm{L}^{*}, \mathrm{a}^{*} \mathrm{y} \mathrm{b}^{*}$ para las formulaciones de merkén analizadas. Se puede observar que en la coordenada $L^{*}$ el valor más alto lo reporta la muestra Merkén UCT005 que presenta diferencias significativas $(\mathrm{p} \leq 0,05)$ con respecto al valor más bajo, representado por la muestra Merkén UCT004. Dichos valores pueden estar condicionados por el porcentaje de ají rojo "Cacho de cabra" proveniente de la Región de La Araucanía presente en dichas formulaciones de merkén. Si se considera el estado de madurez del fruto, estos bajos valores en $L^{*}$ podrían responder de manera similar a lo reportado por Gómez-Ladrón y Pardo-González (1996) donde el valor máximo de L* es alcanzado durante el estado verde-rojo del fruto de Capsicum y disminuye durante el estado final del proceso de 
maduración del ají. Sin embargo, Vega-Gálvez et al. (2009) señalan que la coordenada $\mathrm{L}^{*}$ presenta los valores más bajos a altas temperaturas (90\%), indicando que los ajíes frescos presentan un color más oscuro en comparación con las muestras en seco rehidratadas. Los valores más bajos que corresponden a las muestras Merkén UCT003 y Merkén UCT004 no presentan diferencias significativas ( $>0,05)$, y se caracterizan por presentar mayor porcentaje de ají "Cacho de cabra" proveniente de la Región del Maule en su formulación, esto indica que este tipo de ají produce un color más oscuro en el producto final. Sin embargo, las altas temperaturas de secado podrían estar deteriorando los pigmentos carotenoides (Ergünes y Tarhan, 2006), y la decoloración durante el procesamiento podría ocurrir a través de la oxidación enzimática o no enzimática (Rodríguez-Amaya et al., 2008).

Por otro lado, el Cuadro 3 muestra los resultados obtenidos para el componente verde-rojo (a*) que en la totalidad de las formulaciones predominó la fracción roja, en este sentido Gómez-Ladrón y Pardo-González (1996) señalan que dependiendo del momento de cosecha los valores de a* para las variedades de Capsicum cambian de valores negativos (componente verde) a valores positivos (componente rojo). Sin embargo, las altas temperaturas también tienden a disminuir la coordenada a* (Vega-Gálvez et al., 2009), lo que podría explicarse debido a la presencia de carotenoides, así como también a otros componentes tales como vitaminas, carbohidratos y aminoácidos en pimientos que afectan el color del producto final (Miranda et al., 2009). Los valores medios de $\mathrm{a}^{*}$ presentaron diferencias significativas $(\mathrm{p} \leq 0,05)$ para formulaciones de merkén con mayor contenido de ají procedente de la Región del Maule respecto a aquellas formulaciones que contienen mayor porcentaje de ají "Cacho de cabra" de la Región de La Araucanía. Sin embargo, no se presentan diferencias significativas entre las muestras Merkén UCT001 y Merkén UCT005 elaboradas exclusivamente de ají "Cacho de cabra" de la Región de La Araucanía, siendo la última la que más porcentaje de ají posee en su composición. Esto probablemente se debe a la generación de productos cafés de las reacciones no enzimáticas provocadas por los aumentos de temperatura (Vega-Gálvez et al., 2009). Por otra parte, en un estudio anterior, Ramesh et al. (2001) señalan que los efectos del proceso de secado de los frutos de ají incrementan o disminuyen el contenido de carotenoides en el fruto en la medida que éste aumenta o disminuye, por lo cual se ven condicionados los valores para la fracción roja ( $\left.\mathrm{a}^{*}\right)$. Además, es bien sabido que durante el secado por aire caliente, las verduras sufren cambios físicos, estructurales, químicos y nutricionales que pueden afectar los atributos de calidad como la textura, color, sabor y valor nutricional (Di Scala y Crapiste, 2008).

Los valores medios del componente $b^{*}$ (azulamarillo) mostraron una absoluta inclinación hacia la fracción amarilla en la totalidad de las muestras de merkén analizadas (Cuadro 3). Las formulaciones de merkén elaboradas con mayor porcentaje de ají "Cacho de cabra" de la Región de La Araucanía son las que presentan un mayor valor para la fracción de color amarillo, destacándose Merkén UCT005, que presenta diferencias significativas $(\mathrm{p} \leq 0,05)$ con respecto a Merkén UCT004, elaborado con un mayor porcentaje de ají proveniente de la Región del Maule.

El índice de saturación o cromatismo $\left(\mathrm{C}^{*}\right)$ y tonalidad $\left(\mathrm{H}^{*}\right)$ proporcionan más información acerca de la distribución espacial de los colores que

Cuadro 3. Parámetros de color CIELAB en relación a la formulación del producto.

\begin{tabular}{lccccc}
\hline Formulación & $\mathrm{L}^{*}$ & $\mathrm{a}^{*}$ & $\mathrm{~b}^{*}$ & $\mathrm{C}^{*}$ & $\mathrm{H}^{*}$ \\
\hline Merkén UCT001 & $36,11^{\mathrm{ab}} \pm 0,463$ & $33,21^{\mathrm{a}} \pm 0,553$ & $40,84^{\mathrm{ab}} \pm 0,218$ & $52,73^{\mathrm{ab}} \pm 0,463$ & $50,88^{\mathrm{a}} \pm 0,401$ \\
Merkén UCT002 & $38,6^{\mathrm{ab}} \pm 2,127$ & $31,77^{\mathrm{ab}} \pm 3,145$ & $38,53^{\mathrm{ab}} \pm 3,918$ & $53,89^{\mathrm{ab}} \pm 4,581$ & $50,41^{\mathrm{a}} \pm 2,283$ \\
Merkén UCT003 & $33,25^{\mathrm{ab}} \pm 2,252$ & $27,84^{\mathrm{b}} \pm 0,596$ & $36,87^{\mathrm{ab}} \pm 1,824$ & $46,57^{\mathrm{b}} \pm 1,813$ & $52,87^{\mathrm{a}} \pm 0,784$ \\
Merkén UCT004 & $30,81^{\mathrm{b}} \pm 1,746$ & $27,55^{\mathrm{b}} \pm 0,516$ & $35,21^{\mathrm{b}} \pm 1,389$ & $44,42^{\mathrm{b}} \pm 1,411$ & $51,91^{\mathrm{a}} \pm 0,584$ \\
Merkén UCT005 & $40,69^{\mathrm{a}} \pm 1,523$ & $36,63^{\mathrm{a}} \pm 0,302$ & $45,25^{\mathrm{a}} \pm 0,987$ & $57,27^{\mathrm{a}} \pm 0,953$ & $50,99^{\mathrm{a}} \pm 0,385$ \\
\hline
\end{tabular}

Letras distintas en las columnas difieren significativamente entre tratamientos según Test de Tukey para $\mathrm{L}^{*}$ y $\mathrm{H}^{*}$, Tamhane para $a^{*}$ y Games Howel para $b^{*}$ y $C^{*}(p \leq 0,05)$. Valores en base a la media. $L^{*}$ es luminosidad; $a^{*}$ cromaticidad componente verderojo; b* cromaticidad azul-amarillo; $\mathrm{C}^{*}$ saturación o cromatismo (saturación o pureza del color); $\mathrm{H}^{*}$ tonalidad o coloración de la muestra. 
los valores de las mediciones triestímulas directas, que se definen de acuerdo a la mezcla resultante de los tres colores primarios (Sigge et al., 2001), donde además, ambos parámetros se ven afectados de forma opuesta por la temperatura (Vega-Gálvez et al., 2009). Los valores de $\mathrm{C}^{*} \mathrm{y} \mathrm{H}^{*}$ se muestran en el Cuadro 3, donde se observa que solo $\mathrm{C}^{*}$ se ve afectado $(\mathrm{p} \leq 0,05)$ por la cantidad de ají "Cacho de cabra" de la Región de La Araucanía y de la Región del Maule que respectivamente forman parte en la formulación final del producto. Estos resultados no se relacionarían con lo informado por Gómez-Ladrón y Pardo-González (1996), donde variedades de Capsicum que presentan genes de resistencia de clorofila alcanzan valores más bajos para el parámetro $\mathrm{H}^{*}$ a diferencia de las variedades normales que alcanzan valores más altos para este parámetro.

\section{Conclusiones}

En esta investigación se encontró que existe heterogeneidad del color en las formulaciones de merkén analizadas, a través del contenido de carotenoides y componentes de color Cielab. Esto se relaciona con la procedencia del ají cv. "Cacho de cabra", siendo las formulaciones de merkén elaboradas en base al ají proveniente de la Región de La Araucanía las que presentaron un contenido más alto en carotenoides y componentes de color Cielab, comparado con las formulaciones producidas con ají proveniente de la Región del Maule, cuyos valores fueron más bajos en las variables evaluadas. El grupo de carotenoides amarillo-naranja (A) fue superior al grupo de carotenoides rojos (R) en todas las formulaciones, del mismo modo la fracción de color roja y amarilla determinadas con el método Cielab, destacándose las formulaciones Merkén UCT005 y Merkén UCT001 por presentar los valores más altos para ambos grupos de carotenoides.

Así, de acuerdo con los resultados obtenidos en esta investigación, destacan aquellas formulaciones elaboradas con ají "Cacho de cabra" de la Región de La Araucanía, ya que éstas alcanzaron los valores más altos en el contenido de carotenoides y componentes de color, y, por lo tanto, estos ecotipos de ají cv "Cacho de cabra" son recomendables para una formulación óptima de merkén bajo el punto de vista del color.

\section{Agradecimientos}

Los autores agradecen al proyecto FIA-PIT-2006-1-A-058 por el financiamiento otorgado.

\section{Literatura Citada}

Biacs, P.A.; Czinkotai, B.; Hoschke, A.

1992 Factors affecting stability of colored substances in paprika powders. J. Agric. Food Chem. 40(3): 363-367.

Calvo, C.; Durán, L.

1997 Propiedades físicas II. Ópticas y color. In: Aguilera, J.M. (Ed.). Temas en tecnología de alimentos. Instituto Politécnico Nacional. México. pp. 261-288.

Collera-Zúñiga, O.; García, F.; Meléndez, R.

2005 Comparative study of carotenoid composition in three mexican varieties of Capsicum annuum L. Food Chem. 90: 109-114.

Curl, A.L.

1962 The carotenoids of red bell pepper. J. Agric. Food Chem. 10: 504-509.

Davies, B.H.; Susan, M.; Kirk, J.T.O.

1970 The nature and biosynthesis of the carotenoids of different color varieties of Capsicum annuит. Phytochem. 9: 797-805.

Deepa, N.; Kaur, C.; George, B.; Sing, B.; Kapoor, H. 2007 Antioxidant constituents in some sweet pepper (Capsicum annuит L.) genotypes during maturity. LWT: Food Sci. Technol. 40(1): 121-129.
Di Scala, K.C.; Crapiste, G.H.

2008 Drying kinetics and quality changes during drying of red pepper. LWT: Food Sci. Technol. 41(5): 789-795.

Ergünes, G.; Tarhan, S.

2006 Colour retention of red peppers by chemical pretreatments during greenhouse and open sun dryning. J. Food Eng. 76:446-452.

Fekete, M.; Kozma, L.; Huszka, T.

1976 Spectrophotometric method for determining the pigment content of ground paprika. Zeitschrift für LebensmittelUntersuchung und-Forschung 161: 31-33.

García, M.I.; Lozano, M.; Montero, V.; Ayuso, M.C.; Bernalte, M.J.; Vidal-Aragón, M.C.; Pérez, M.M.

2007 Agronomic characteristics and carotenoid content of five Bola-type paprika red pepper (Capsicum annuum L.) cultivars. Scientia Horticulturae: 113: 202-207.

Gómez-Ladrón, R.; Pardo-González, J.

1996 Evolution of color during the ripening of selected varieties of paprika pepper (Capsicum annuиm L.). J. Agric. Food Chem. 44: 2049-2052.

Haspel-Horvatovic, E.; Horickova, B.

1976 Spectrophotometrical determination of paprika-pigments. Zeitschrift für Lebensmittel-Untersuchung und-Forschung 160: 275-276. 
Hornero-Méndez, D.; Gómez-Ladrón, R.; Mínguez-Mosquera, M.I. 2000 Carotenoids biosynthesis change in five red pepper (Capsicum annuиm L.) cultivars during ripening. Cultivar selection for breeding. J. Agric. Food Chem. 48: 3857-3864. INE

2008 Resultados preliminares del VII Censo Agropecuario. Disponible en http://www.censoagropecuario.cl/noticias/07/11/13112007.htlm. Leído el 11 de septiembre de 2008.

Méndez-Trujillo, V.; González-Mendoza, D.; Gutiérrez-Miceli, F.A. 2005 Contenido de carotenoides y color extractable de nuevos cultivares en chile pimiento. Revista Chapingo. Serie Horticultura 11(2): 215-218.

Mendoza, F.; Aguilera, J.M.

2004 Application of image analysis for classification of ripening bananas. J. Food Sci. 69(9):E471-E477.

Mínguez-Mosquera, M.I.; Pérez-Gálvez, A.; Hornero-Méndez, D. 2005 Pigmentos carotenoides en frutas y vegetales; mucho más que simples colorantes naturales. CTC Alimentación $\mathrm{N}^{\circ} 26$ p. 108-113.

Mínguez-Mosquera, M.I.; Gandul-Rojas, B.

1994 Mechanism and kinetics of carotenoid degradation during the processing of green table olives. J. Agric. Food Chem. 42: 1551-1554.

Miranda, M.; Maureira, H.; Rodríguez, K.; Vega-Gálvez, A.

2009 Influence of temperature on the drying kinetics, physicochemical properties, and antioxidant capacity of aloe vera (Aloe barbadensis Miller) gel. J. Food Eng. 91(2): 297-304.
Ramesh, M.N.; Wolf, W.; Tevini, D.; Jung, G.

2001 Influence of processing parameters on the drying of spice paprika. J. Food Eng. 49: 63-72.

Rodriguez-Amaya, D.B.; Kimura, M.; Godoy, H.; Amaya-Farfan, J. 2008 Updated Brazilian database on food carotenoids: Factors affecting carotenoid. J. Food Compos. Anal. 21: 445-463.

Russo, V.M.; Howard, L.R.

2002 Carotenoids in pungent and non-pungent peppers at various developmental stages grown in the field glasshouse. J. Sci. Food Agric. 82: 615-624.

Sigge, G.O.; Hansmann, C.F.; Jourbert, E.

2001 Effect of storage conditions, packaging material and metabisulfite treatment on the colour of dehydrated green bell peppers (Capsicum annuиm L.). J. Food Qual. 24: 205-218.

Thomet, M.; Sepúlveda, J.

2006 Estudio de factibilidad para la promoción y comercialización del merkén de Lumaco. Programa Araucanía Tierra Viva. Lumaco, Chile. 81 pp.

Topuz, A.; Ozdemir, F.

2007 Assessment of carotenoids, capsaicinoids and ascorbic acid composition of some selected pepper cultivars (Capsicum annuит L.) grown in Turkey. J. Food Compos. Anal. 20: 596-602.

Vega-Gálvez, A.; Di Scala, K.; Rodríguez, K.; Lemus-Mondaca, R.; Miranda, M.; López, J.; Perez-Won, M.

2009 Effect of air-drying temperature on physico-chemical properties, antioxidant capacity, colour and total phenolic content of red pepper (Capsicum annuum, L. var. Hungarian). Food Chem. 117: 647-653. 